\title{
Triple reuptake inhibitor potentially ameliorates the chronic unpredictable stress induced comorbid depression and anxiety: A Behavioural based rodent test battery evaluation
}

\author{
Dilip Kumar Pandey*1, Baldev Gautam², Ankur Jindal ${ }^{2}$, Shvetank Bhatt ${ }^{2}$ \\ ${ }^{1} / n$-vivo Pharmacology, NDDD, Lupin Research Park, Pune, Maharashtra, India \\ ${ }^{2}$ Pharmacy Department, FD-III, Birla Institute of Technology \& Science, Pilani, Rajasthan-333031, India.
}

\begin{abstract}
Depression and anxiety tend to be the most prevalent conditions among the multitude of neurobehavioural disorders which cause distress in chronic stress condition. The objective of the present investigation was to examine the effect of DOV-216303 (a tripe re-uptake inhibitor) in depression-like and anxiety-like behaviour of rats following CUS. Rats were subjected to an experimental setting of CUS for 20 days. Escitalopram (10 mg/ $\mathrm{kg}$ and DOV-216303 $(2.5,5$ and $10 \mathrm{mg} / \mathrm{kg})$ were administered to stressed and sham rats for 14 days during stress period from day 7 of stress induction. Following the CUS and treatment, rats were subjected to a rodent's behavioural test battery (depression/anxiety). The results demonstrate the depression-like behaviour of CUS rats in modified open field exploration, socio-sexual interaction. In addition, an anxiety-like behaviour was evident in social interaction and elevated plus-maze exploration paradigms. Chronic treatment with Escitalopram (10 mg/ $\mathrm{kg}$ ) and DOV-216303 (5 and $10 \mathrm{mg} / \mathrm{kg}$ ) significantly attenuated the CUS associated behavioural deficits. In conclusion, the aforesaid behavioural anomalies observed in CUS rats are analogous to comorbid anxiety and depression in humans.
\end{abstract}

Key words: DOV-216303, Comorbidity, CUS, Depression, Anxiety, Behavioural test Battery.

\section{INTRODUCTION}

The term "comorbidity" has often been a mystery and is a bottleneck in the antidepressant and anxiolytic drug developments. Comorbidity is usually referred as the co-occurrence (or dual diagnosis) of two disorders or syndromes in the same patient, regardless of whether the disorders are co-incidentally or casually linked ${ }^{1}$. Comorbidity is also well-known for the epidemiologic linkage between two disorders in which one share the pathologic mechanisms over the other ${ }^{2,3}$. The diagnostic and statistical manual of mental disorder (DSM-IV) includes a category of mixed anxiety and depressive disorder, which is the association of sub-threshold depressive symptoms and sub-threshold anxiety when neither symptoms of anxiety nor symptoms of depression (when considered separately) are severe enough for the diagnosis of a disorder to be justified. Nearly half of depressed patients have anxiety symptoms and vice-versa ${ }^{1}$ depression exacerbated by anxiety is associated with a much higher suicide rate than that in the general nondepressed population. ${ }^{4}$ Neuro-biochemical evidence also suggests that both conditions are related to disturbances in serotonergic, dopaminergic and nor-adrenergic transmission and regulation. ${ }^{5}$ Chronic unpredictable stress (CUS) lead depression and anxiety also witness the change in level of BDNF and corticosterone. Brain-derived-neurotrophic factor (BDNF) is one of several
Submission Date: 04/06/2014 Revision Date : :27/08/2014 Accepted Date : :23/12/2014

DOI: 10.5530/ijper.49.1.7 Correspondence Address Dr. Dilip Kumar Pandey, M.Pharm, Ph.D In-vivo Pharmacology, NDDD, Lupin Research Park, Pune, Maharashtra, India Mo: 91+7875533880, Mail: pandeysd11408@gmail. com

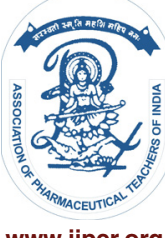

www.ijper.org 
endogenous proteins that play a critical role in the survival, maintenance, and growth of central and peripheral neurons. Depressed patients with comorbid anxiety disorders have a inferior prognosis; are less responsive to therapy and may encounter greater deficits in psychosocial task as compared with depressed patients without comorbid anxiety disorders. ${ }^{5,6}$ Most antidepressant currently in use increases the synaptic biogenic amine with majority of drug selectively inhibiting the reuptake of serotonin and norepinephrine. ${ }^{7}$ These compounds include serotonin and norepinephrine (dual) reuptake inhibitors such as venlafaxine and milnacipran, serotonin-selective reuptake inhibitors including citalopram, fluoxetine and paroxetine, and norepinephrine-specific reuptake inhibitors such as reboxetine. Nevertheless, monotherapy with selective 5-HT reuptake inhibitors leaves room for improvement, as approximately $30 \%$ of patients do not respond to treatment and many more show only a partial therapeutic response. ${ }^{8}$ The application of selective serotonin re-uptake inhibitors (SSRIs) in the depression therapy is well known, (fluoxetine). There are various issues with the use of SSRIs: latency of onset (2-3 weeks), lack of efficacy (approximately $30 \%$ non-responders) and unwanted side effects for instance sexual dysfunction, sleep disturbances, nausea, anxiety and reduced appetite. Further a newer approach to address some of these concerns has been the development of SNRIs (Serotonin and Nor-epinephrine reuptake inhibitors) for example venlafaxine. Recently, so-called triple inhibitors, which additionally block the dopamine transporter was evacuated. ${ }^{7}$ Previously it has been shown that DOV 216,303 (a tripe re-uptake inhibitor) has antidepressant-like effects in the forced swim test (both rats and mice) and the mouse tail suspension test (Skolnick et al, 2003) as well as in humans."

In the current study, DOV 216,303 has been evaluated for the chronic CUS induced comorbid depression and anxiety. This particular model is thought to mimic the everyday, low-grade yet unexpected stressors often experienced by humans and that can trigger depressive episodes, compromising physical and psychological health. Previously, using Traumatic brain injury model, comorbid depression anxiety has been evaluated in rats. ${ }^{10}$ In CUS, chronic stress resulted in neurochemical neurobehavioural changes resembling those of depressed and anxiety patients. The association between stress, depression and anxiety has long been observed, particularly at the clinical level, where chronic exposure to stressful life events has been associated with the development of depressive and anxious symptoms in certain individuals, under certain stress full conditions. ${ }^{11,12}$ Most CUS may be related to the dysfunction of serotonergic neurotransmission in the prefrontal cortex and limbic system, although involvement for glutamatergic system have also been reported. ${ }^{13,14}$ Several lines of evidence suggest that, depletion of monoamines : serotonin, noradrenaline and dopamine sustained stress could be the reason for anxiety and behavioral depression. ${ }^{13}$

\section{MATERIALS AND METHODS}

\section{Animals}

Male Wistar rats (180-220 g) were obtained from Hissar Agricultural University, Haryana, India. All animals were maintained under standard laboratory conditions [12 hour light/dark cycle temperature $22 \pm 2^{\circ} \mathrm{C}$; relative humidity; $60 \pm 5 \%$ in the Central Animal Facility. Rats were given sterilized food (standard pellet chow feed) and filtered water ad libitum. Following a quarantine period of two weeks the animals were randomly assigned to different experimental groups.

\section{Ethical Approval}

The experiments on animal were performed in accordance with the protocol approved by the Institutional Animal Ethics Committee (IAEC) of Birla Institute of Technology \& Science, Pilani, India.

\section{Drugs and Chemicals}

Escitalopram (ESC) and DOV-216303 [(士)-1-(3,4-dichlorophenyl)-3-azabicyclo-[3.1.0] hexane. $\mathrm{HCl}$ ] (a Triple re-uptake inhibitor, from DOVE Pharmaceutical) were obtained from Glenmark Research Laboratories, India and DOVE Parma, USA. As generous gift samples. The Escitalopram and DOV-216303 were dissolved distilled water. The doses of all drugs were selected on the basis of preliminary testing.

\section{Biochemical analysis}

\section{Serum corticosterone level}

To evaluate the change in the HPA axis, we measured serum corticosterone level. Measurement of serum corticosterone was performed using a commercially available enzyme-linked immunosorbent assay (ELISA) kit (IBL, USA) according to the manufacturers' instructions. All samples were measured in duplicate.

\section{Measurement of BDNF Concentration}

BDNF concentration was measured using commercially available ELISA kits. Briefly, microtiter plates (96-well) were coated for 90 minute with the samples diluted 1:10 in sample diluents. The plate content was discarder after 90 minutes. Then a biotinylated anti-rat BDNF antibody diluted 1:100 in antibody diluents buffer was added to each well and incubated for $1 \mathrm{~h}$ at room 
temperature. After this the plate was washed three times with washing buffer. After washing, an Avidin-BiotinPeroxidase working solution diluted 1:100 with ABC dilution buffer was added to each well and incubated at room temperature for 30 minutes. After 30 minutes the plate was washed five times with washing buffer. After this TMB colour developing agent was added in each well and incubated for 20 minutes in dark. Then reaction stop solution was added and the amount of BDNF was determined by absorbance at $450 \mathrm{~nm}$. The standard curve demonstrates a direct relationship between optical density and BDNF concentration.

\section{Pharmacological Procedures}

\section{General Considerations for Behavioural Studies}

The drug administrations and behavioural screenings were performed between 0900 and $1700 \mathrm{hrs}$ or as per the design of behavioural test. The drugs solutions were freshly prepared in distilled water and administered p.o. (as specified) in a constant volume of $10 \mathrm{ml} / \mathrm{kg}$ before experiment. The drug/vehicle treated animals were acclimatized to the experimentation room for one hour before testing.

\section{Schedule for Drug administration and Behavioural tests}

The schedule for behavioural tests and drug administration as shown in Table 1 and Table 2. The admin-

\begin{tabular}{|c|c|c|}
\hline Day & Schedule of Stressor & Time \\
\hline 1 & Cold water stress $\left(8^{\circ} \mathrm{C}\right)$ & $5 \mathrm{~min}$ \\
\hline 2 & Tail Pinching & $1 \mathrm{~min}$ \\
\hline 3 & Food and water deprivation & $24 \mathrm{hr}$ \\
\hline 4 & Swimming $\left(24 \pm 2^{\circ} \mathrm{C}\right.$ water $)$ & $20 \mathrm{~min}$ \\
\hline 5 & Overnight illumination & $12 \mathrm{hr}$ \\
\hline 6 & No stress & ----- \\
\hline 7 & Tail pinching & $1.5 \mathrm{~min}$ \\
\hline 8 & Cold water swim $\left(10^{\circ} \mathrm{C}\right)$ & $5 \mathrm{~min}$ \\
\hline 9 & Swimming $\left(24 \pm 2^{\circ} \mathrm{C}\right.$ water $)$ & $15 \mathrm{~min}$ \\
\hline 10 & Tail pinching & $2 \mathrm{~min}$ \\
\hline 11 & Cold water swim $\left(6^{\circ} \mathrm{C}\right)$ & $5 \mathrm{~min}$ \\
\hline 12 & Swimming $\left(24 \pm 2^{\circ} \mathrm{C}\right.$ water $)$ & $15 \mathrm{~min}$ \\
\hline 13 & Tail pinching & $2 \min$ \\
\hline 14 & Over night illumination & $12 \mathrm{hr}$ \\
\hline 15 & Cold water swim $\left(6^{\circ} \mathrm{C}\right)$ & $5 \min$ \\
\hline 16 & No stress & ---- \\
\hline 17 & Food and water deprivation & $24 \mathrm{hr}$ \\
\hline 18 & Tail pinching & $1.5 \mathrm{~min}$ \\
\hline 19 & Swimming $\left(24 \pm 2^{\circ} \mathrm{C}\right.$ water $)$ & $15 \mathrm{~min}$ \\
\hline 20 & Over night illumination & $12 \mathrm{hr}$ \\
\hline 21 & Cold water swim $\left(6^{\circ} \mathrm{C}\right)$ & $5 \mathrm{~min}$ \\
\hline 22 & Rest day & ------- \\
\hline
\end{tabular}

istration of DOV-216303 (2.5, 5 and $10 \mathrm{mg} / \mathrm{kg})$ and ESC $(10 \mathrm{mg} / \mathrm{kg} / 10 \mathrm{ml}$ p.o, daily) were started from day 7 - 21. After 14 days of treatment or vehicle, the first behavioural test was performed. One hour before the behavioural assessment, the rats were acclimatized to testing room, which was free from any noise.

The behavioural testing was done post $24 \mathrm{hr}$ of the last dose in order to evade the acute effect of treatment. Drug treatment was continued till $22^{\text {nd }}$ day of the study. Behavioural models, incorporating repeated exposure to stress have been widely used as experimental models for depression ${ }^{12}$ as stress is thought to play an important role in the etiology of depression and anxiety.

\section{Experimental Design}

In this model animal(s) were divided into sham and CUS groups.

\begin{tabular}{|l|l|}
\hline Sham + Control $=6$ & CUS + Control $=6$ \\
\hline $\begin{array}{l}\text { Sham + DOV-216303 } \\
(2.5 \mathrm{mg} / \mathrm{kg})=6\end{array}$ & CUS + DOV-216303 \\
\hline Sham + DOV-216303 & CUS + DOV $/ \mathrm{kg})=6$ \\
$(5 \mathrm{mg} / \mathrm{kg})=6$ & $(5 \mathrm{mg} / \mathrm{kg})=6$ \\
\hline Sham + DOV-216303 & CUS + DOV-216303 \\
$(10 \mathrm{mg} / \mathrm{kg})=6$ & $(10 \mathrm{mg} / \mathrm{kg})=6$ \\
\hline Sham + ESC $(10 \mathrm{mg} / \mathrm{kg})=6$ & CUS + ESC $(10 \mathrm{mg} / \mathrm{kg})=6$ \\
\hline
\end{tabular}

\section{Induction of Chronic Unpredictable stress (CUS)}

The CUS procedure was performed as cited in the literature. ${ }^{12}$ This animal model of stress consists of chronic exposure to various mild social and environmental stressors, none of which are sufficient alone to induce long-lasting effects. The stressors used were varied and applied in a different sequence each week (3 weeks) to avoid any habituation. The CUS procedure was applied for 21 consecutive days is shown in Table-1. This paradigm was devised to maximize unpredictability; in that the stressors were applied in seemingly random order and at varying times during the light phase (0900-1700 $\mathrm{hr})$. The procedures were carried out in isolated rooms adjacent to the rat housing unit, requiring minimal handling or transport of the rats. After each stressor, animals were kept in a recovery room for $1-2 \mathrm{hr}$, following which they were placed in clean cages with fresh bedding and returned to the housing facility. Sham rats were individually housed for the same period of time and were handled daily for 30s in the housing room, but were not stressed. Animal(s) with abnormal behaviour were excluded from the study. All the animals were given uniform stress throughout the procedure. All the rats were subjected to stress so as to induce a traumatic insult to brain region involved in the regulation of depression and anxiety-like condition. Details of stressors are given in Table-1. Post 21 days of study, blood samples were collected in anti-coagulant containing sterile centrifuge 
Table 2: Schedule of Treatments and Behavioural Assessments on CUS/ Sham Rats

\begin{tabular}{|c|c|c|c|}
\hline Day 1-21 & Day 7-21 & \multicolumn{2}{|c|}{ Behavioural Test Schedule } \\
\cline { 2 - 3 } & & Day 23 $^{\text {rd }}$ & Day 24 $^{\text {th }}$ \\
\hline Chronic & $\begin{array}{c}\text { All the drugs } \\
\text { and vehicle were } \\
\text { administered } \\
\text { once a } \\
\text { day for 14 days }\end{array}$ & Open field test (D) & Social interaction test (A) \\
\cline { 2 - 4 } & maze test (A) & $\begin{array}{c}\text { Socio- sexual interaction } \\
\text { Test (D) }\end{array}$ \\
\end{tabular}

tubes and stored at $-70^{\circ} \mathrm{C}$ until the estimation of corticosterone and BDNF.

\section{Behavioural Assays Performed Post-Traumatic Chronic Unpredictable Stress in Rats}

\section{Open Field Test}

The OFT was conducted as described by Kelly ${ }^{15}$ with slight modifications. The apparatus consisted of a circular (90-cm diameter) arena with $75-\mathrm{cm}$ high polished metal walls and floor equally divided into $10 \mathrm{~cm}$ squares. A $60 \mathrm{~W}$ light bulb was positioned $90 \mathrm{~cm}$ above the base of the arena which was the only source of illumination in the testing room. Each animal was individually placed in the center of the open field apparatus and the following parameters were observed for $5 \mathrm{~min}$.

Ambulation scores (number of squares crossed) and number of rearing episodes were noted as horizontal and vertical activity, respectively. Crossing of a square was considered only when the hind limbs of the animal moves to the next square. Other parameter observed was defecation. The number of fecal pellets was counted at the end of each $5 \mathrm{~min}$. trial. The apparatus was cleaned with ethyl alcohol and dried between trials to remove any residual odour.

Rationale: Measurement of hyperactivity reflects psychomotor effect.

\section{Elevated Plus Maze}

The procedure was adopted same as described by Yamada. ${ }^{16}$ The plus-maze consisted of two open $(50 \mathrm{~cm}$ $\mathrm{x} 10 \mathrm{~cm})$ arms and two enclosed $(50 \mathrm{~cm} \times 10 \mathrm{~cm})$ arms surrounded by $30-\mathrm{cm}$ high walls. The four arms were joined by a central platform $(10 \mathrm{~cm} \times 10 \mathrm{~cm})$ open to all the arms, to form a plus shape.

The entire apparatus was elevated to a height of $60 \mathrm{~cm}$ above the floor. The apparatus was indirectly illuminated with a ceiling mounted lamp $(60 \mathrm{~W})$ which was placed $90 \mathrm{~cm}$ above the apparatus. At the beginning of the test, the animal was placed in the central platform facing an open arm. The number of time rats enter the open and closed arms and time spent in open arms were recorded for $5 \mathrm{~min}$. open arm duration, i.e., the total amount of time spent by the rat in an open arm; closed arm duration, i.e., the total amount of time spent by the rat in a closed arm; open arm entries, i.e., the total number of entries with all four paws in the open unprotected arms; closed arm entries, i.e., the total number of entries with all four paws in the closed protected arms. After each test, the apparatus was sprayed with dilute alcohol and wiped thoroughly to eliminate the residual odour.

Rationale: Natural aversion to open area, preference of protected and unprotected area.

\section{Socio-Sexual Interaction Test}

The present protocol was adapted from that of Bergeron $^{17}$, Meyerson and Hoglund ${ }^{18}$ and performed with a slight modification. The apparatus for the Sociosexual interaction test consisted of a transparent Plexiglas box $[45 \mathrm{~cm}(\mathrm{~L}) \times 30 \mathrm{~cm}(\mathrm{~W}) \times 40 \mathrm{~cm}(\mathrm{H})]$ with a black wooden base, illuminated with a red lamp (10 W). $1 \mathrm{~cm}$ of husk layer was made over the wooden base. Socio-sexual behaviour was observed from 22:00 to 3:00 $\mathrm{h}$ in the dark phase of the illumination cycle. A male rat was first placed into the Plexiglas box to be habituated to the environment for $5 \mathrm{~min}$. Then, a sexually receptive female rat which had received subcutaneous injections of $0.14 \mathrm{mg}$ of estradiol 72 and $48 \mathrm{~h}$ before the test and $0.7 \mathrm{mg}$ of progesterone $4 \mathrm{~h}$ before the test. The sexual behaviour of the male rats was observed for $40 \mathrm{~min}$ following 5 min of male rat acclimatization. The following parameters of socio-sexual interaction behaviour were recorded: starting latency of genital-probing (licking and grooming female rats) and thrusting, number of genital-probing and thrusting and pursuit (duration of male rat following female rats) by male rat. After each test, the apparatus was sprayed with alcohol and wiped thoroughly to eliminate the residual odour.

Rationale: loss of desire was measured.

\section{Social Interaction Test}

The protocol mentioned elsewhere ${ }^{19}$ was adapted with slight modification as mentioned below. The same apparatus and testing environment as those of the open field test were used for the social interaction test, except that the illumination was milder $(15 \mathrm{~W})$ than that of the open field test. On the day of operation, pairs of rats of the same group housed in different cages were put into 
two different corners of the open field arena. The social interaction behaviour including the passive interaction (number of crossing to each other) and social interaction time (s) (grooming, mounting and crawling under the other rat) were recorded for 5 min after placing the rats in the apparatus. After each test, the apparatus was sprayed with dilute alcohol and wiped thoroughly to eliminate residual odour.

Rationale: Simulation of human social behaviour.

\section{Statistical analysis}

All analysis was performed using graph pad prism 5 for windows. All the results of experiments are expressed as mean \pm S.E.M. Statistical differences were evaluated with a one-way analysis of variance (ANOVA) followed by the bonferroni for multiple comparisons. The criterion for a statistically significant difference was fixed to $\mathrm{p}<0.05$.

\section{RESULTS}

\section{Effect of CUS on Body Weight}

Figure 1. displays the effect of CUS on body weight of rats. Body weight of sham and CUS rats was continuously observed till the behavioural tests were started. Decrease in body weight was observed in CUS rats. Body weight gained was significanlty lesser in CUS rats compared to the sham rats (Figure 1.). Treatment induced change was not observed in the body weight of sham and CUS rats.

\section{Open Field Exploration}

CUS rats exhibited hypo-locomotion in OFT. CUS rats showed decreased ambulation $\left[\mathrm{F}_{(9,50)}=3.42, \mathrm{P}<0.05\right)$ and rearing $\left.\mathrm{F}_{(9,50)}=3.33, \mathrm{P}<0.05\right]$ and increased fecal pellets $\left[\mathrm{F}_{(9,50)}=0.94, \mathrm{P}<0.05\right]$ behaviour for $5 \mathrm{~min}$ after being put into the open field arena (Table-3). The results of this study demonstrated that, both decreased frequencies of ambulation and rearing were significantly

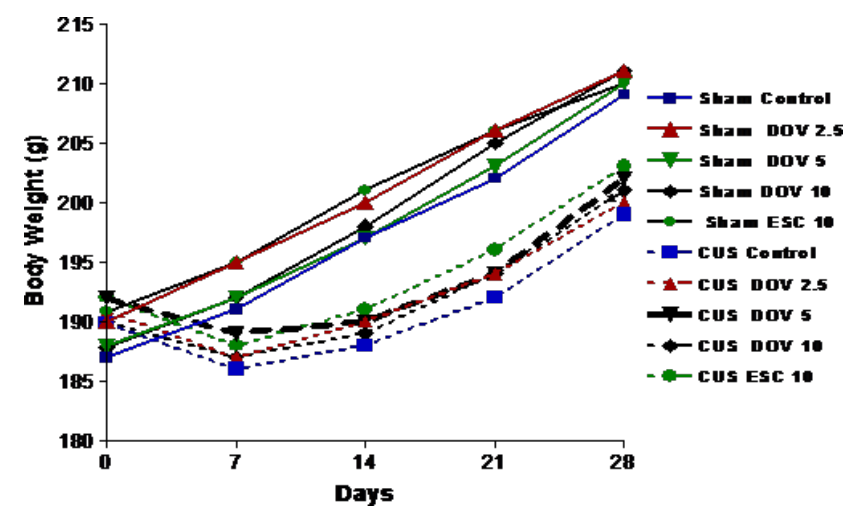

Figure 1 : Effect of CUS on change in body weight. Figure represents the mean change in body weight. All drugs/vehicle $(\mathrm{mg} / \mathrm{kg})$ were administered $\mathrm{p}$. 0 . once a day for 14 days. $* \mathrm{P}<0.05$ vs sham control. $\mathrm{n}=6$ per group reversed by chronic DOV-216303 (5 and $10 \mathrm{mg} / \mathrm{kg}$ ) and ESC $(10 \mathrm{mg} / \mathrm{kg})$ treatment. Additionally, DOV-216303 significantly reduced the number of fecal pellets. However, the ambulation and rearing in the sham group were not significantly affected by the treatment with DOV216303.

\section{Socio-Sexual interaction test}

Socio-sexual behaviour and the effect of DOV-216303 (5 and $10 \mathrm{mg} / \mathrm{kg}$ ) was examined in socio-sexual interaction test in CUS rats. The starting latency of genital probing $\left[\mathrm{F}_{(9,50)}=10.01, \mathrm{P}<0.05\right]$ and thrusting $\left[\mathrm{F}_{(9,50)}=\right.$ 3.7, $\mathrm{P}<0.05$ ] in CUS rats was significantly increased, whereas the number of genital probing $\left[\mathrm{F}_{(0,50)}=3.17\right.$, $\mathrm{P}<0.05]$, thrusting events $\left[\mathrm{F}_{(9,50)}=4.7, \mathrm{P}<0.05\right]$ and pursuit $\left[\mathrm{F}_{9,50}=3.5, \mathrm{P}<0.05\right)$ were decreased as compared to sham rats (Table-4). CUS rats showed significant socio-sexual deficits in the socio-sexual interaction test indicating a core symptom of depression. Chronic treatment with DOV-216303 (5 and $10 \mathrm{mg} / \mathrm{kg}$ ) and ESC (10 $\mathrm{mg} / \mathrm{kg})$ significantly $(\mathrm{P}<0.05)$ improved the socio-sexual behaviour exhibited by CUS rats, without affecting the behaviour in sham rats.

\section{Elevated Plus Maze Test}

The time spent and number of entries in open arms by sham and CUS rats, cumulated over the 5-min test was displayed (Figure.2). CUS rats exhibited a significant $(\mathrm{P}<0.05)$ reduction in the percent entries and time spent in the open arms as compared to sham rats. These results are indicative of an enhanced anxiety response in rats exposed to stress compared with sham control rats. Chronic DOV-216303 (5 and $10 \mathrm{mg} / \mathrm{kg}$ ) and ESC treatment $(10 \mathrm{mg} / \mathrm{kg})$ significantly increased the percent entry $\left[\mathrm{F}_{(9,50)}=13.10, \mathrm{P}<0.05\right]$ and time spent $\left[\mathrm{F}_{(9,50)}=\right.$ 8.1, $\mathrm{P}<0.05]$ in open arms compared to CUS control in EPM task (Figure 2). Also DOV-216303 and ESC significantly increased the number of entries and time spent in the open arms by sham rats.

\section{Social Interaction Test}

Duration of social interaction time and number of passive interactions were recorded in social interaction test after being put in open field for $5 \mathrm{~min}$. As demonstrated in Figure 3 unfamiliar pairs of CUS rats showed decreased social interactive time compared with sham rats $\left[\mathrm{F}_{(9,50)}=7.27, \mathrm{P}<0.05\right]$. In addition, $\mathrm{CUS}$ rats showed increased passive interaction $\left[\mathrm{F}_{(9,50)}=7.4, \mathrm{P}<0.05\right] \mathrm{com}$ pared to sham rats in social interaction test (Fig.3). The social impairment in CUS rats was significantly $(\mathrm{P}<0.05)$ ameliorated by the chronic DOV-216303 $(5-10 \mathrm{mg} / \mathrm{kg})$ and ESC $(10 \mathrm{mg} / \mathrm{kg})$ treatment. The treatment with DOV-216303 and ESC enhanced the duration of inter- 


\section{Table 3: Effect of ESC on Open Field Behaviour of Sham and CUS rats}

\begin{tabular}{|c|c|c|c|}
\hline Treatment (mg/kg) & No. of Ambulation & No. of Rearing & No. of Fecal pellets \\
\hline \multicolumn{4}{|l|}{ Sham } \\
\hline Control & $96.00 \pm 4.44$ & $11.33 \pm 1.62$ & $2.80 \pm 0.79$ \\
\hline DOV-216303 (2.5) & $99.83 \pm 6.93$ & $13.00 \pm 2.08$ & $3.00 \pm 0.81$ \\
\hline DOV-216303 (5) & $100.66 \pm 10.28$ & $14.00 \pm 1.12$ & $2.53 \pm 0.71$ \\
\hline DOV-216303 (10) & $110.66 \pm 7.70$ & $14.50 \pm 1.80$ & $2.33 \pm 0.43$ \\
\hline ESC 10 & $107.30 \pm 11.48$ & $13.67 \pm 1.12$ & $2.17 \pm 0.70$ \\
\hline \multicolumn{4}{|l|}{ CUS } \\
\hline Control & $69.83 \pm 8.08^{*}$ & $5.16 \pm 1.78^{*}$ & $4.83 \pm 0.82^{*}$ \\
\hline DOV-216303 (2.5) & $72.67 \pm 8.59^{*}$ & $6.50 \pm 1.52^{*}$ & $3.50 \pm 0.76^{*}$ \\
\hline DOV-216303 (5) & $92.50 \pm 8.26^{\#}$ & $12.60 \pm 2.13^{\#}$ & $2.50 \pm 1.12^{\#}$ \\
\hline DOV-216303 (10) & $105.16 \pm 8.24^{\#}$ & $12.83 \pm 1.9^{\#}$ & $2.66 \pm 1.09^{\#}$ \\
\hline ESC 10 & $93.33 \pm 11.07^{\#}$ & $11.33 \pm 1.23^{\#}$ & $2.83 \pm 0.79^{\#}$ \\
\hline
\end{tabular}

\section{Table 4: Effect of CUS and Drug Treatment on Socio-Sexual Behaviour in Socio-Sexual Interaction Test in Rats}

\begin{tabular}{|c|c|c|c|c|c|}
\hline Treatment (mg/kg) & $\begin{array}{l}\text { Genital Probing } \\
\text { Latency (Sec) }\end{array}$ & $\begin{array}{l}\text { No. of Genital } \\
\text { Probing }\end{array}$ & $\begin{array}{c}\text { Thrusting Latency } \\
\text { (Sec) }\end{array}$ & $\begin{array}{l}\text { Number of } \\
\text { Thrusting }\end{array}$ & Pursuit (min) \\
\hline \multicolumn{6}{|l|}{ Sham } \\
\hline Control & $12.67 \pm 2.2 .5$ & $35.00 \pm 5.26$ & $23.0 \pm 4.05$ & $36.83 \pm 4.55$ & $2.43 \pm 0.25$ \\
\hline DOV-216303 (2.5) & $12.33 \pm 3.22$ & $35.66 \pm 4.80$ & $24.33 \pm 5.95$ & $37.0 \pm 3.80$ & $2.51 \pm 0.35$ \\
\hline DOV-216303 (5) & $12.17 \pm 2.65$ & $36.33 \pm 4.58$ & $24.83 \pm 2.51$ & $36.66 \pm 4.60$ & $2.46 \pm 0.25$ \\
\hline DOV-216303 (10) & $11.50 \pm 4.14$ & $38.00 \pm 5.87$ & $20.17 \pm 2.50$ & $38.33 \pm 3.88$ & $2.73 \pm 0.28$ \\
\hline ESC (10) & $12.33 \pm 2.05$ & $35.84 \pm 5.18$ & $21.33 \pm 4.69$ & $35.40 \pm 5.46$ & $2.58 \pm 0.35$ \\
\hline \multicolumn{6}{|l|}{ CUS } \\
\hline Control & $40.67 \pm 3.99^{*}$ & $12.40 \pm 2.33^{*}$ & $58.67 \pm 13.51^{*}$ & $15.60 \pm 1.83^{*}$ & $1.05 \pm 0.12^{*}$ \\
\hline DOV-216303 (2.5) & $36.00 \pm 5.93^{*}$ & $14.33 \pm 2.65^{\star}$ & $53.17 \pm 14.79^{*}$ & $20.00 \pm 2.90^{*}$ & $1.03 \pm 0.12^{*}$ \\
\hline DOV-216303 (5) & $20.50 \pm 3.15^{\#}$ & $27.00 \pm 5.93^{\#}$ & $34.67 \pm 9.14^{\#}$ & $32.66 \pm 3.77^{\#}$ & $2.20 \pm 0.42^{\#}$ \\
\hline DOV-216303 (10) & $16.67 \pm 2.88^{\#}$ & $30.20 \pm 7.29^{\#}$ & $24.02 \pm 4.94^{\#}$ & $38.70 \pm 3.04^{\#}$ & $2.40 \pm 0.41^{\#}$ \\
\hline ESC (10) & $17.83 \pm 2.80^{\#}$ & $27.33 \pm 5.81^{\#}$ & $52.83 \pm 7.94^{*}$ & $21.60 \pm 3.63^{*}$ & $2.20 \pm 0.42^{\#}$ \\
\hline
\end{tabular}

action and decreased the passive interaction of sham rats.

\section{Effect of CUS and DOV-216303 on Adrenal Gland Weight of Rats}

CUS significanlty $(\mathrm{P}<0.05)$ increased the adrenal gland weight of rats compared to sham group. The chronic treatment with DOV-216303 and escitaloram ( $10 \mathrm{mg} /$ $\mathrm{kg})$ significanltly $\quad\left[\mathrm{F}_{(0,50)}=24.4, \quad \mathrm{P}<0.05\right)$ reversed the increased adrenal weight as compared to stressed group (Figure 4).

\section{Effect of CUS on the level of Corticosterone and BDNF}

Figure 5 shows the calibration curve of corticosterone. CUS rats showed significant $\left[\mathrm{F}_{(9,50)}=6.6, \mathrm{P}<0.05\right]$ increase in the serum corticosterone levels as com- pared to sham rats. The chronic treatment with DOV$216303(5-10 \mathrm{mg} / \mathrm{kg})$ and ESC $(10 \mathrm{mg} / \mathrm{kg})$ significanltly reversed the increased corticosterone level as compared to stressed group.

\section{Serum BDNF concentration}

Figure 6 illustrates the effect of the DOV-216303 $(2.5,5 \& 10 \mathrm{mg} / \mathrm{kg}$, p.o.) and ESC (10 mg/kg, p.o.) on serum BDNF level. Stressed rats showed a significant decrease in serum BDNF level as compared to sham control rats $(\mathrm{p}<0.05)$. Chronic treatment with DOV$216303\left(5-10 \mathrm{mg} / \mathrm{kg}\right.$, p.o.) significantly $\mathrm{F}_{(9,50)}=19.11$, $\mathrm{p}<0.05]$ increased the serum BDNF level in stressed rats as compared to vehicle treated stressed rats. ESC treatment $(10 \mathrm{mg} / \mathrm{kg}$, p.o.) also significantly $(\mathrm{p}<0.05)$ increased serum BDNF level in stressed rat. On the 

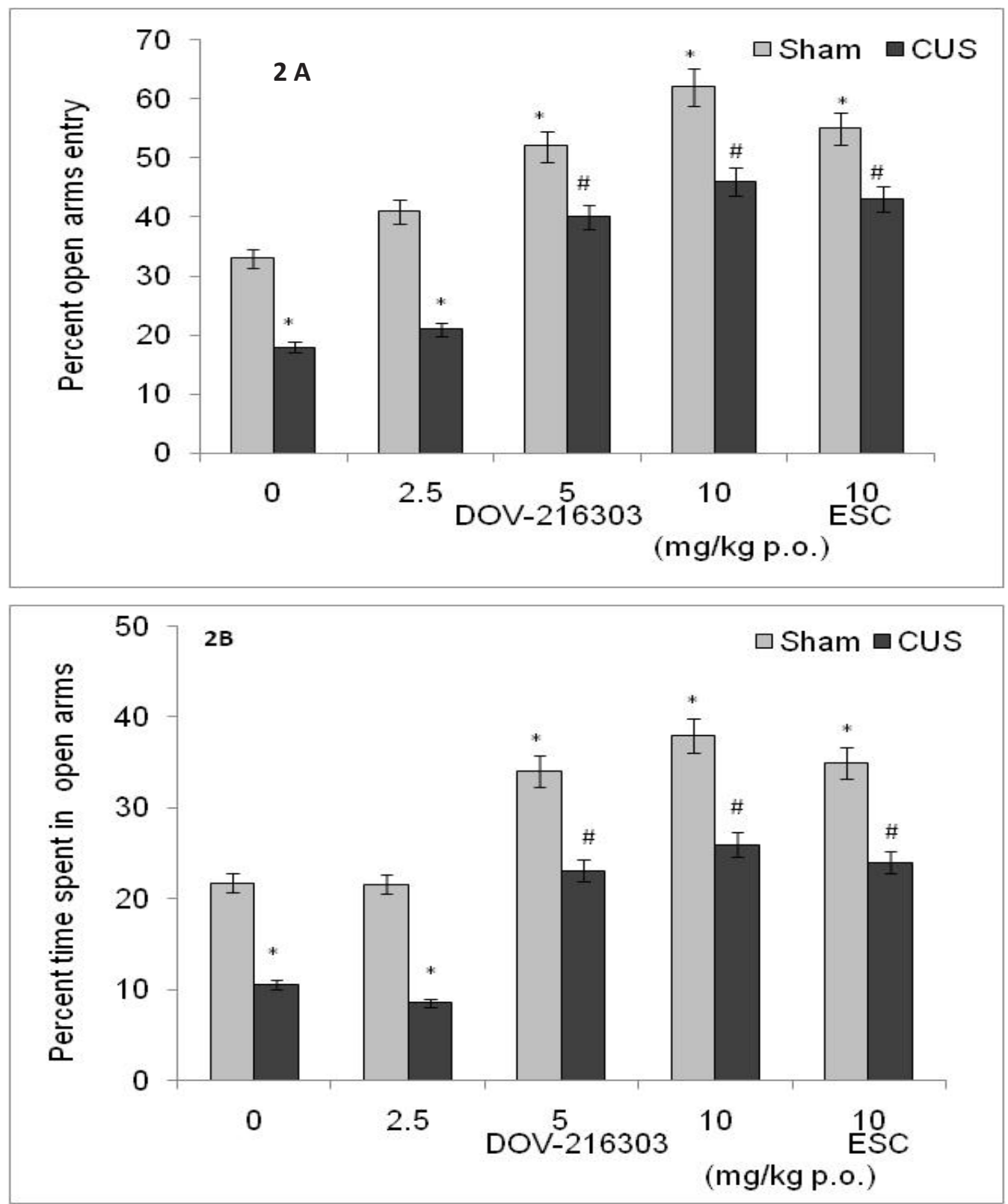

Figure 2 : Effect of DOV-216303 $(2.5-10 \mathrm{mg} / \mathrm{kg})$ and ESC $(10 \mathrm{mg} / \mathrm{kg})$ on the percent entry and time spent in open arms of sham and CUS rats in EPM test.

All the drugs/vehicle were administered p.o. once day for 14 days.The column bar represent mean percent open arm entry. Error bars represent mean S.E.M. *P < 0.05 vs sham control, \#P<0.05 vs CUS control. $n=6$ per group

other hand, DOV-216303 and ESC treatment had no effect on serum BDNF level in sham control rat.

\section{DISCUSSION}

The current study demonstrated that, exposure to chronic unpredictable stress developed the expression of depression and anxiety-like behaviours in rats. Diverse stressors activate a wide spectrum of interacting neuronal and hormonal systems that guide behavioral and physiological responses, leading to alterations in behavior, autonomic function and the secretion of multiple hormones including corticosterone. Clinical studies have shown that stressful life experiences are important etiological factors in the development and maintenance of depression and affective disorders. ${ }^{20,21,22}$ Formerly, hierarchical and segregated concept of depression and anxiety are now viewed as overlapping entities. ${ }^{23}$ In addition, high degree of comorbid (co-occurrence) depression and anxiety are observed in depressed human patients and the margins between depression and anxiety have become blurred..$^{24} \mathrm{~A}$ variety of stress situations have been employed to investigate the consequences of stress and to evaluate the depression and anxiety-like behaviours in rats. As such, a workable model of experimental stress has to incorporate the factors of chronicity and unpredictability of stressors. The experimental design of the current study 

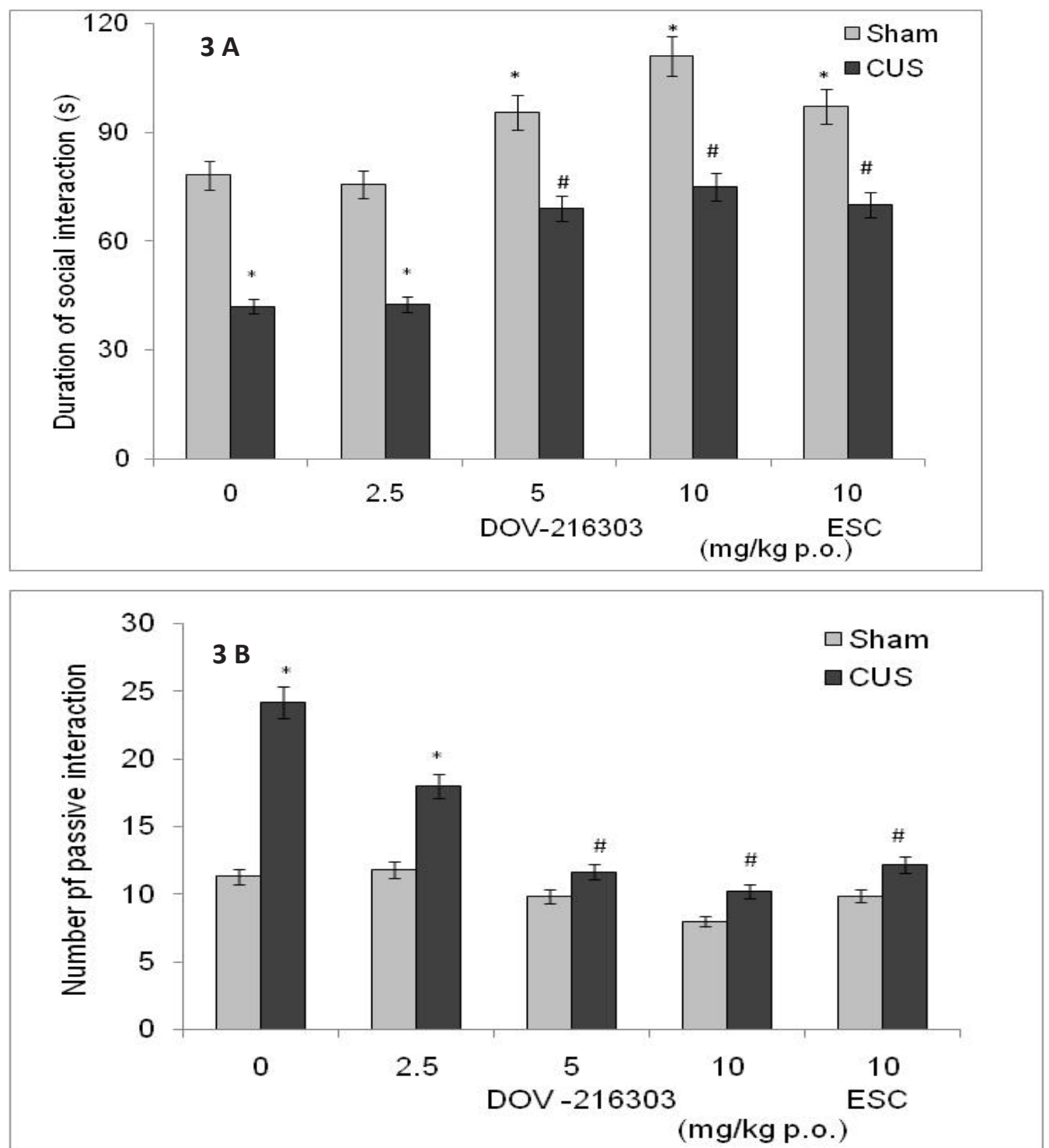

Figure 3 : Effect of DOV-216303 $(2.5-10 \mathrm{mg} / \mathrm{kg})$ and ESC $(10 \mathrm{mg} / \mathrm{kg})$ on the interaction time and number of passive interaction of sham and CUS rats in social interaction test.

All the drugs/ vehicle were administred p.o. once a day for 14 days. The column bar represent mean number of passive interaction. Error bars represent mean S.E.M. *P< 0.05 vs sham control, \#P<0.05 vs to CUS control. $\mathrm{n}=6$ per group

fulfils these criteria to induce significant behavioural deficits in rats. Effect of ESC and DOV-216303 was evaluated in CUS induced mixed anxiety and depression in rats. DOV-216303 is the prototype of a class of compounds referred to as "triple" re-uptake inhibitors. Such compounds inhibit the re-uptake of nor-adrenaline, serotonin and dopamine, the three neurotransmitters most closely linked to major depressive disorder. ${ }^{25,7}$

\section{Behavioural Deficits in CUS Rats Resembling the Symptoms of Depression}

Persistent exposure to uncontrollable and dissimilar aversive situations leads to behavioural alterations in response to subsequent novel stressors. ${ }^{11,26}$ The concept of a stress induced depression has recently been proposed in light of new findings from animal and human studies. Depression associated with stress involves a number of body systems such as the neuroendocrine and neurotransmitter system as well as the immune system including cytokines and the dysregulation of the HPA axis interacting in complex pathways. CUS causes a temporary suppression of food intake and reduction in body weight of stressed rats as compared to sham rats. ${ }^{27}$

Open field exploration investigates the stress induced behaviour $^{28}$ characterized by ambulation, rearing and defecation. When the rats were placed in a novel environment, similar paradigm was observed in CUS rats. CUS rats exhibited reduced locomotor activity in open field test which may possibly mimic some aspects of human psychomotor retardation ${ }^{29}$, an accompanying symptom of major depression in humans. ${ }^{30}$ Normal rats generally show increased rearing in a novel open field, 


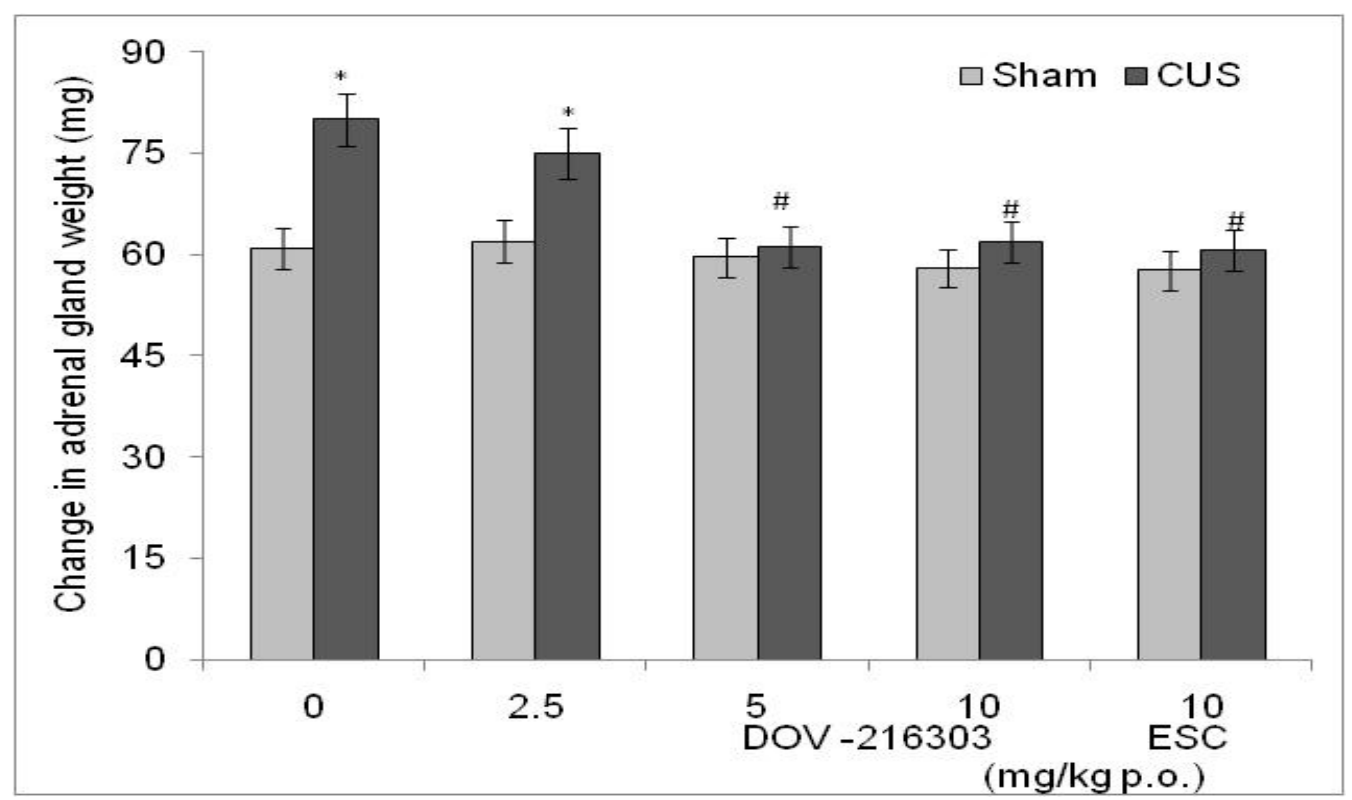

Figure 4 : Effect of DOV-216303 $(2.5-10 \mathrm{mg} / \mathrm{kg})$ and ESC $(10 \mathrm{mg} / \mathrm{kg})$ on adrenal weight of sham / CUS rats. Data represents the mean change in adrenal weight. All the drugs/vehicle were administered p.o. once a day for 14 days. Error bars represent mean S.E.M. *P $<0.05$ Vs sham control, $\# \mathrm{P}<0.05$ vs CUS control. $\mathrm{n}=6$ per group

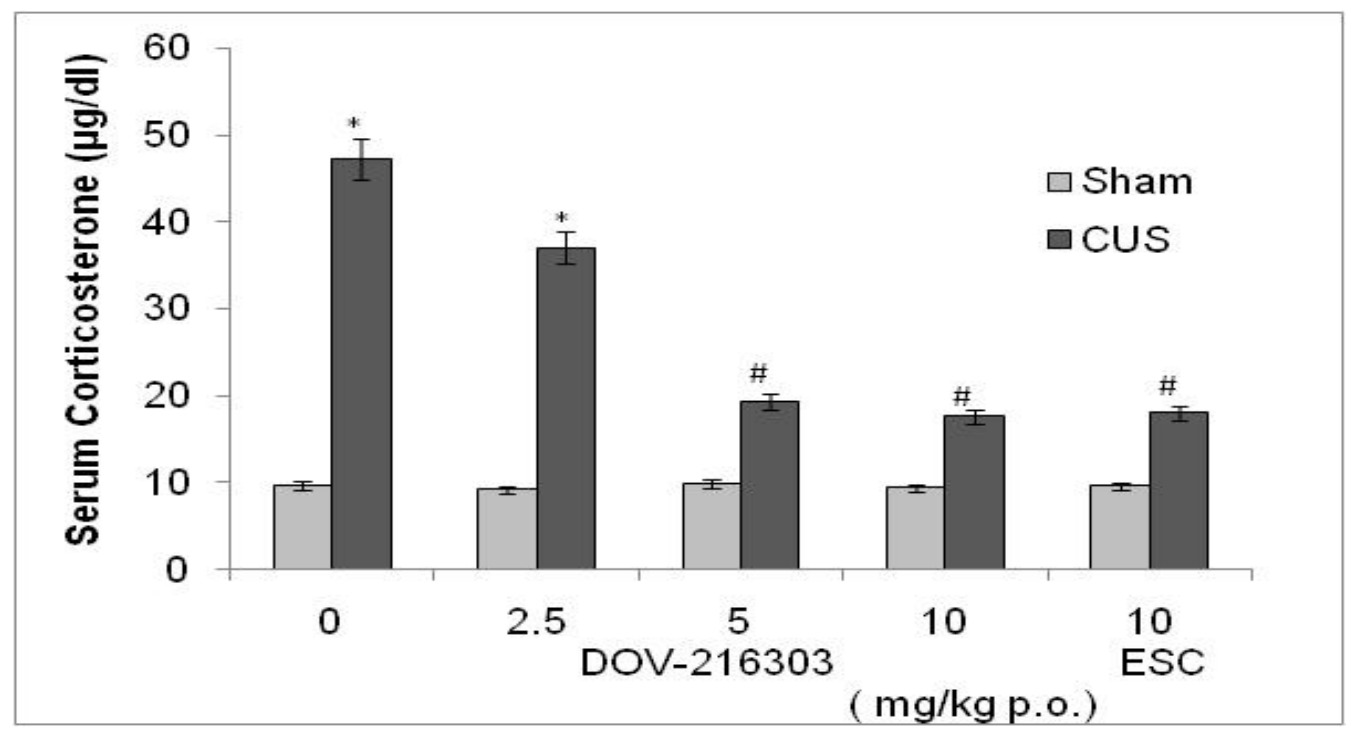

Figure 5 : Effect of CUS and drug treatment on the serum corticosterone levels in sham and CUS rats.

All the drugs /vehicle were administered p.o. once day for 14 days. Each column represent mean corticosterone level. Error bars represent mean S.E.M. *P < 0.05 Vs sham control, \#P< 0.05 vs CUS control. $n=6$ per group.

whereas CUS rats display decreased rearing and crossings in a novel open field. The chronic DOV-216303 and ESC treatment significantly normalized the CUS induced behavioural deficits in open field exploration. Through in this test, psychomotor retardation was successfully modeled in CUS rats. Further the additional parameter observed in CUS rats was socio-sexual behavior. Stress can lead to diminished sexual desire. The loss of sexual interest is defined as a core symptom of depression which was a successfully modeled in rat. ${ }^{31,32,33}$ The decreased libido in CUS rats adds to the validation of using this model of depression in the current study. The results of this study explains that, chronic sequential exposure to a variety of stressors causes a substantial decrease in socio-sexual interaction (sexual anhedonia) as indicated by increased latency in genital probing and thrusting and decreased episodes of genital probing, thrusting and pursuits which resembles the loss of interest in socio-sexual behaviour, a core symptom of depression. Socio-sexual behaviour was successfully modeled in this test. The behavioural deficit was effectively normalized by chronic treatment with traditional anti-depressant ESC and DOV-216303.

\section{Behavioural Deficits in CUS Rats Resembling the Symptoms of Anxiety}

Research work done earlier has explained that exposure to stress can augment anxiety in humans. ${ }^{34,35}$ The tests 


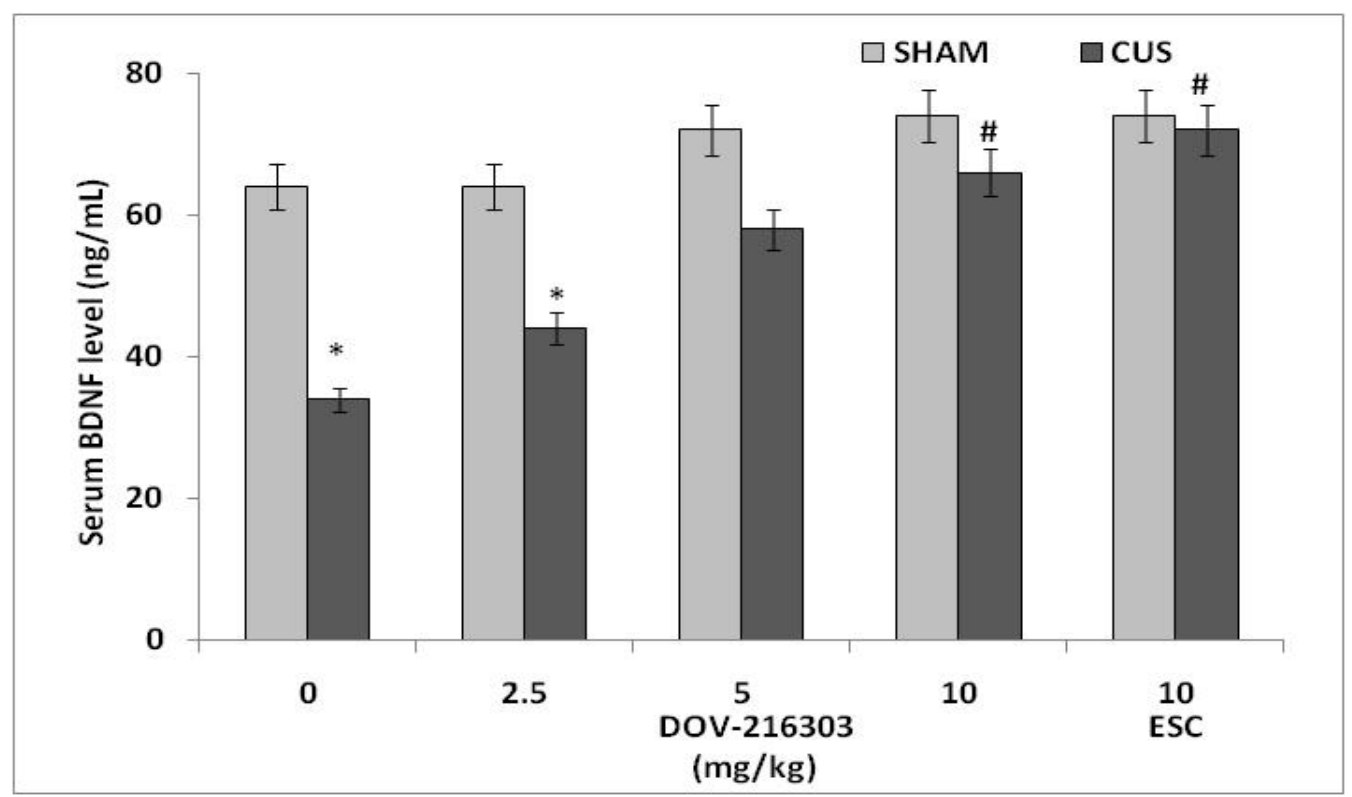

Figure 6 : Effect of CUS and drug treatment on the serum BDNF levels in sham and CUS rats.

All the drugs/vehicle were administered p.o. once day for 14 days. Each column represent mean corticosterone level. Error bars represent mean $\mathrm{S}$. E.M. $* \mathrm{P}<0.05 \mathrm{vs}$ sham control, $\# \mathrm{P}<0.05$ vs CUS control. $\mathrm{n}=6$ per group.

used here can be differentiated and described as a model of "social anxiety" (assessed in the social exploration test in rats), a model of anxiety in an "approach-avoidance conflict" (assessed in the elevated plus maze).

In addition to the depression, CUS exposure also produced a consistent increase in anxiety-like behavior. The EPM test has been extensively used for screening of anxiolytic drugs and for exploring neurobiological basis of anxiety ${ }^{36,37}$ and the classical indices of anxiety-related behaviour is the number of open arm entries and the time spent on the open arms. ${ }^{38,39}$ The current study demonstrated that, CUS prolonged the time spent in closed arm and decreased the number of entries to open arm. EPM results suggest that chronic stressed rats, behave more anxiously than sham control rats. Decreased time spent and entry in open arm may be due to the fear of open arm and increased serum corticosterone. It has been suggested that reluctance to explore open arm is caused by fear of open arm rather than the height of the maze. ${ }^{37}$ The fear of open arm is mediated by the amygdala itself which is affected by the CUS. This may explain the increase in stress-induced anxiety-like behavior on the elevated plus-maze. These results are in accordance with the previous finding using these signs as stress markers. ${ }^{40}$ The results confirm the anxiogenic-like effect of stress in EPM. DOV-216303 and ESC significantly reversed the CUS induced anxiety like behaviour.

The social interaction test is used for the simulation of social traits in rodents. Impaired social interaction is an indicative test of heightened level of anxiety. ${ }^{19}$ Chronic stressor produces limitation in the ability to perform social roles and may interfere with social interaction. ${ }^{41}$ Decreased social interaction among the rats indicates the anxiety-like ${ }^{19}$ and depression-like symptoms. The most important finding of the current study is impaired social interaction as indicated by diminished social interaction time and increased passive interaction in CUS rats. The results suggested that, these animals are more anxious/ fearful due to anticipated stressful event. Chronic DOV216303 and ESC treatment, significantly increased social interaction and decreased passive interaction in CUS rats similar to that seen in sham group. Further, CUS induced behavioural deficits were associated with the serum concentration of corticosterone and adrenal weight, as both correlate with the stress effect. ${ }^{42}$ In the present study, CUS significantly increased the adrenal weight and serum corticosterone. Elevated serum corticosterone could be due to repeated chronic stress leading to the disruption of glucocorticoid negative feed back system ${ }^{43}$ which is thought to be the important factor to develop CUS induced comorbid depression and anxiety (Figure 5).

The recent hypotheses of CUS and the mechanism of action of antidepressants include alterations of the neurotrophic factors, particularly of BDNF. Reduction in BDNF shows the chronic stress-induced depression and anxiety disorders. The ability of BDNF to cross the blood-brain barrier suggests that blood BDNF level may directly reflect BDNF levels in the brain. Chronic, but not acute, SSRI treatment by increasing 5-HT neurotransmission causes an increase in BDNF expression. Moreover, the majority of studies report decreased serum BDNF levels in depressive patients, suggesting 
that blood BDNF levels could serve as useful biological marker for improving depression treatment. ${ }^{43}$ Antidepressant, increasing the level of neurotransmitter like 5-HT, stimulates the expression of BDNF, and BDNF enhances the growth and survival of monoamine neurons. Hence, we assessed the serum BDNF protein level that may be mediating the therapeutic effects of DOV-216303 in the CUS model. In this investigation, CUS rats showed a significant decrease in serum BDNF level as compared to the sham control rats. Treatment with DOV-216303 reversed the CUS-induced alteration in the serum BDNF level, which suggested the action mechanism for the antidepressant-and anxiolytic-like effect of DOV-216303.

The chronic drug treatment reversed the CUS induced neuro-endocrinal changes. Although the precise mechanism of CUS induced behavioural deficits remain unclear, the involvement of serotonergic and dopaminergic dysfunction in pre-frontal cortex and hippocampus following chronic stress ${ }^{44}$ are implicated in depression and anxiety. The current data do not directly address the question of which particular neural areas are involved in CUS. However, limbic areas like the hippocampal sub-fields, lateral septum and central amygdala are particularly vulnerable to damage.

Though the availability of several types of antidepressant and anxiolytic drugs are more, but their is steady rise in the prevalence of depression and anxiety due to stressed life style..$^{45,46}$ The present study constitute an extensive behavioural data characterizing the effect of CUS and action of escitalopram, on behavioural features resembling the sequelae of psychiatric comorbidity following CUS. The CUS for 21 days resulted in the development of comorbid depression and anxiety-like behaviour. Escitalopram and DOV-216303, a Triple re-uptake inhibitor significantly reversed the behavioural deficits induced by CUS. The effectiveness of DOV-216303 in CUS induced comorbid condition was increased due to addition of the dopamine component to serotonin and nor-adrenaline transporter blockade, but further studies are needed to substantiate this point.

\section{CONCLUSION}

Owing to the role of the neurotransmitters serotonin,noradrenaline and dopamine in depression and anxiety, the triple reuptake inhibitor DOV-216303 was evaluated in above mentioned CUS associated disorder. In view of the fact that animal models and predictive tests symbolize only one or few of the symptoms of psychiatric disorders which are commonly revised and their pathogenesis being revisited, it is necessary to conduct neuro- chemical investigations to purport CUS rat as a model of comorbid depression and anxiety and to examine the molecular mechanism of DOV-216303 in CUS induced depression and anxiety.

\section{CONFLICT OF INTEREST}

Authors confirms no conflict of interest.

\section{ACKNOWLEDGEMENT}

The authors wish to thank Glenmark Research Centre, Mumbai for providing drug sample. Authors would also like to thank the Council of Scientific and Industrial Research, New Delhi, India for partly funding this work.

\section{REFERENCES}

1. Feinstein AR. The pre-therapeutic classification of comorbidity in chronic disease. Journal of Chronic Diseases 1970; 23(7): 455-68.

2. Kessler RC, Nelson CB, McGonagle KA, Liu J, Swartz M, Blazer DG. Comorbidity of DSM-III-R major depressive disorder in the general population: results from the US National Comorbidity Survey. British Journal of Psychiatry 1996; 168(30): 17-30.

3. Krishnan R, Delong M, Kraemer H, Carney R, Spiegel D, Gordon C. Comorbidity of depression with other medical diseases in the elderly. Biological Psychiatry 2002; 52(6): 559-88.

4. Nutt DJ, Ballenger JC, Sheehan D, Wittchen HU. Generalized anxiety disorder: comorbidity, comparative biology and treatment. International Journal of Neuropsychopharmacology 2002; 5(04): 315-25.

5. Roy-Byrne PP, Stang P, Wittchen HU, Ustun B, Walters EE, Kessler RC. Lifetime panic depression comorbidity in the National Comorbidity Survey. Association with symptoms, impairment, course and help-seeking. British Journal of Psychiatry 2000; 176(3): 229-35.

6. Kessler RC, Chiu WT, Demler O. Prevalence, severity, and comorbidity of 12-month DSM-IV disorders in the National Comorbidity Survey Replication. Archives of General Psychiatry 2005; 62(6): 617-27.

7. Skolnick P, Popik P, Janowsky A, Beer B, Lippa AS. Antidepressant-like actions of DOV 21, 947: a "triple" reuptake inhibitor. Eur. J. Pharmacol. 2003; 46: 99-104.

8. Angst J. Major depression in 1998: are we providing optimal therapy? J. Clin. Psychiatry. 1999; 60(6): 5-9.

9. Skolnick P, Krieter P, Tizzano J, Basile A, Popik P, Czobor P, Lippa A. Preclinical and clinical pharmacology of DOV 216, 303, a "triple" reuptake inhibitor. CNS Drug Rev. 2006; 12(2): 123-34.

10. Pandey DK, Yadav SK, Mahesh R, Rajkumar R. Depression-like and anxietylike behavioural aftermaths of impact accelerated traumatic brain injury in rats: A model of comorbid depression and anxiety? Behavioural Brain Research 2009; 205(2): 436-42.

11. Katz RJ, Roth KA, Carroll BJ. Acute and chronic stress effects on open field activity in the rat: Implications for a model of depression. Neuroscience Biobehavioural Reviews 1981; 5(2): 247-51.

12. Willner P. Validity, reliability and utility of the chronic mild stress model of depression: a 10-years review and evaluation. Psychopharmacology (Berl) 1997; 134(4): 319-29.

13. Li JM, Kong LD, Wang YM, Cheng CH, Zhang WY, Tan WZ. Behavioral and biochemical studies on chronic mild stress models in rats treated with a Chinese traditional prescription Banxiahoupu decoction. Life Science 2003; 74(1): 55-73.

14. Beck K, Luine V. Food deprivation modulates chronic stress effects on object recognition in male rats: role of monoamines and amino acids. Brain Research 1999; 830(1): 56-71.

15. Kelly JP, Wrynn, AS, Leonard BE. The olfactory bulbectomized rat as a model of depression: an update. Pharmacology and Therapeutics 1997; 74(3): 299305. 
16. Yamada K, lida R, Miyamoto Y, Saito K, Sekikawa K, Seishima M. Neurobehavioural alterations in mice with a targeted deletion of the tumor necrosis factor- $\alpha$ gene: implications for emotional behaviour. Journal of Neuroimmunology 2000; 111(1): 131-8.

17. Breigeiron MK, Morris M, Lucion AB, Sanvitto GL. Effects of angiotensin II microinjected into medial amygdala on male sexual behaviour in rats. Hormone and Behaviour 2002; 41(3): 267-74.

18. Meyerson BJ, Hoglund AU. Exploratory and socio-sexual behaviour in the male laboratory rat: A methodological approach for the investigation of drug action. Acta Pharmacology Toxicology 1981; 48(2): 168-80.

19. File SE, Hyde JR. Can social interaction be used to measure anxiety? British Journal of Pharmacology 1978; 62(1): 19 -24.

20. Kendler KS, Heath A, Martin NG, Eaves LJ. Symptoms of anxiety and depression in a volunteer twin population: The etiologic role of genetic and environmental factors Archives of General Psychiatry 1986; 43(3): 213-20.

21. Kendler KS, Kessler RC, Walters EE, MacLean C, Neale MC. Stressful life events, genetic liability, and onset of an episode of major depression in women. American Journal of Psychiatry 1995; 152(6): 837-41.

22. Mineka S, Watson D, Clark LA. Comorbidity of anxiety and unipolar mood disorders. Annual Review of Psychology 1998; 49(1): 377-86.

23. Levine J, Cole DP, Chengappa KN, Gershon S. Anxiety disorders and major depression. together or apart, Depression Anxiety 2001; 14(2): 94-106.

24. Gorman JM. Comorbid depression and anxiety spectrum disorders. Depression Anxiety 1996; 4(4): 160-8.

25. Cryan JF, Markou A, Lucki I. Assessing antidepressant activity in rodents: recent developments and future needs, Trends in Pharmacological Science 2002; 23(5): 238-45

26. Willner P. The validity of animal models of depression. Psychopharmacology 1984; 83(1): 1-6.

27. Lennie TA, McCarthy DO, Keesey RE. Body energy status and the metabolic response to acute inflammation. American Journal of Physiology 1995; 269(5): R1024-30.

28. Denenberg $\mathrm{VH}$. Open-field behavior in the rat: what does mean? Annals of the Newyork Academy of Science 1969; 159(3): 852-8.

29. D'Aquila PS, Brain P, Willner P. Effects of chronic mild stress on performance in behavioural tests relevant to anxiety and depression, Physiology and Behaviour 1994; 56(5): 861-7.

30. Willner P, Towell A, Sampson, D Sophokleous S, Muscat R. Reduction of sucrose preference by chronic unpredictable mild stress, and its restoration by a tricyclic antidepressant, Psychopharmacology 1987; 93(3): 358-64.

31. Muruganandam AV, Kumar V, Bhattacharya SK. Effect of poly herba formulation, Eumil, on chronic stress-induced homoeostatic perturbations in rats. Indian Journal of Experimental Biology 2002; 40(10): 1151-6.

32. Bhattacharya A, Ghosal S, Bhattacharya SK. Antioxidant effect of Withania somnifera glycowithanolides in chronic foot shock stress-induced perturbations of oxidative free radical scavenging enzymes and lipid peroxidation in rat frontal cortex and striatum. Journal of Ethanopharmacology 2001; 74(1): 1-6.

33. Edwards DA, Griffis KT, Tardivel C. Olfactory bulb removal: effects on sexual behaviour and partner-preference in male rats. Physiology and Behaviour 1990; 48(3): 447-50.

34. Regier DA, Narrow WE, Rae DS, Manderscheid RW, Locke BZ, Goodwin FK. The de facto mental and addictive disorders service system. Epidemiologic Catchment Area prospective 1-year prevalence rates of disorders and services. Archives of General Psychiatry 1983; 50(02): 85-92.

35. Koba T, Kodam Y, Shimizu K, Soichiro N, Sugawara M, Kobayashi Y. Persistent behavioural changes in rats following inescapable shock stress: a potential model of posttraumatic stress disorder. World Journal of Biological Psychiatry 20001; 2(1): 34-42.

36. Rex A, Voigt JP, Gustedt C, Beckett S, Fink H. Anxiolytic-like profile in Wistar, but not Sprague-Dawley rats in the social interaction test. Psychopharmacology (Berl) 2004; 177(1-2): 23-34.

37. Pellow S, Chopin P, File SE. Validation of open: closed arm entries in elevated plus maze as a measure of anxiety in rats. Journal of Neuroscience 1985; 14(3): 149-54

38. Rodgers RJ, Cole JC, Aboualfa K, Stephenson LH. Ethopharma- cological analysis of the effects of putative "anxiogenic" agents in the mouse elevated plus maze. Pharmacology Biochemistry and Behavior 1995; 52: 1-6.

39. Andersen IL, Boe KE, Foerevik G, Janczak AM, Bakken M. Behavioural evaluation of methods for assessing fear responses in weaned pigs. Applied Animal Behaviour Science 2000; 69(3): 227-32.

40. Magariños MA, McEwen BS. Stress-induced atrophy of apical dendrites of hippocampal CA3c neurons: involvement of glucocorticoid secretion and excitatory amino acid receptors. Neuroscience 1995; 69(1): 89-94.

41. Blazer DG. Social support and mortality in an elderly community population. American Journal of Epidemiology 1982; 115(5): 684-90.

42. Sapolsky RM, Romero LM, Munck AU. How do glucocorticoids influence stress responses? Integrating permissive, suppressive, stimulatory, and preparative actions. Endocrine Review 2000; 21(1): 55-60.

43. Carroll BJ, Feinberg M, Greden JF, Tarika J, Albala AA, Haskett RF, et al. A specific laboratory test for the diagnosis of melancholia: Standardization, validation and clinical utility. Archives of General Psychiatry 1981; 38(1): 1521.

44. Mizoguchi K, Yuzurihara M, Ishige A, Sasaki H. Chronic stress differentially regulates glucocorticoid negative feedback response in rats. Psychoneuroendocrinology 2001; 26(5): 443-8.

45. Andrews G, Sanderson K, Slade T, Issakidis C. Why does the burden of disease persist? Relating the burden of anxiety and depression of effectiveness of treatment. Bulletin of WHO. 2000; 78(4): 446-54.

46. Kessler RC. The effects of stressful life events on depression. Annals of Review Psychology 1997; 48: 191-7. 